\title{
Zagreb Eccentricity Indices of the Generalized Hierarchical Product Graphs and Their Applications
}

\author{
Zhaoyang Luo ${ }^{1,2}$ and Jianliang $\mathrm{Wu}^{1}$ \\ ${ }^{1}$ School of Mathematics, Shandong University, Jinan 250100, China \\ ${ }^{2}$ Department of Mathematics, Changji University, Changji 831100, China \\ Correspondence should be addressed to Jianliang Wu; jlwu@sdu.edu.cn
}

Received 12 October 2013; Accepted 22 December 2013; Published 10 February 2014

Academic Editor: Juan Manuel Peña

Copyright (c) 2014 Z. Luo and J. Wu. This is an open access article distributed under the Creative Commons Attribution License, which permits unrestricted use, distribution, and reproduction in any medium, provided the original work is properly cited.

Let $G$ be a connected graph. The first and second Zagreb eccentricity indices of $G$ are defined as $M_{1}^{*}(G)=\sum_{v \in V(G)} \varepsilon_{G}^{2}(v)$ and $M_{2}^{*}(G)=$ $\sum_{u v \in E(G)} \varepsilon_{G}(u) \varepsilon_{G}(v)$, where $\varepsilon_{G}(v)$ is the eccentricity of the vertex $v$ in $G$ and $\varepsilon_{G}^{2}(v)=\left(\varepsilon_{G}(v)\right)^{2}$. Suppose that $G(U) \sqcap H(\emptyset \neq U \subseteq V(G))$ is the generalized hierarchical product of two connected graphs $G$ and $H$. In this paper, the Zagreb eccentricity indices $M_{1}^{*}$ and $M_{2}^{*}$ of $G(U) \sqcap H$ are computed. Moreover, we present explicit formulas for the $M_{1}^{*}$ and $M_{2}^{*}$ of $S$-sum graph, Cartesian, cluster, and corona product graphs by means of some invariants of the factors.

\section{Introduction}

A topological index is a real number associated with chemical constitution purporting for correlation of chemical structure with various physical properties, chemical reactivity, or biological activity, which is used to understand properties of chemical compounds in theoretical chemistry [1].

Up to now, hundreds of topological indices have been defined in chemical literatures, various applications of these topological indices have been found, and many mathematical properties are also investigated. Wiener index $W$ is the first topological index, introduced by American chemist Wiener, for investigating boiling points of alkanes in 1947 [2]. The well known degree-based topological indices are the first and second Zagreb indices $M_{1}$ and $M_{2}$, which have been introduced by Gutman and Trinajstić [3] and applied to study molecular chirality in quantitative structure-activity relationship (QSAR) and quantitative structure-property relationship (QSPR) analysis and so forth. Resently, the first and second Zagreb eccentricity indices $M_{1}^{*}$ and $M_{2}^{*}$ have been introduced by Ghorbani and Hosseinzadeh [4] and Vukičević and Graovac [5] as the revised version of the Zagreb indices $M_{1}$ and $M_{2}$, respectively. They computed the Zagreb eccentricity indices of some composite graphs and showed that $M_{1}^{*}(G) /|G| \geq M_{2}^{*}(G) /|E(G)|$ holds for all acyclic and unicyclic graphs and that neither this nor the opposite inequality holds for all bicyclic graphs. For further results of the Zagreb eccentricity indices, we encourage the reader to refer to [6-8].

In 2009, Spain mathematicians Barrière and coauthors [9] introduced a new composite graph, namely, hierarchical product graph. In the same year, this team also reported a generalization of both Cartesian and the hierarchical product of graphs, namely, the generalized hierarchical product of graphs in [10]. After that, many results for some topological indices of the (generalized) hierarchical product of graphs are reported; see [11-17].

In this paper, the Zagreb eccentricity indices of the generalized hierarchical product graph $G(U) \sqcap H$ are computed and as some special cases of $G(U) \sqcap H$, the Zagreb eccentricity indices of the Cartesian product graph $G \square H$, the $S$-sum graph $G+{ }_{S} H$, and the cluster product graph $G\{H\}$ are determined, respectively. Moreover, as applications, we present explicit formulas for the $M_{1}^{*}$ and $M_{2}^{*}$ indices of the $C_{4}$ nanotorus $C_{m} \square C_{n}$, the $C_{4}$ nanotubes $P_{m} \square C_{n}$, the zig-zay polyhex nanotube $\operatorname{TUHC}_{6}[2 n, 2]$, the hexagonal chain $L_{n}$, and so forth.

\section{Preliminaries}

Throughout this paper, all graphs are simple, finite, and undirected. For terminology and notations that are not defined here, we refer the reader to West [18]. 
Let $G=\left(V(G), E(G), \psi_{G}\right)$ be a graph with the vertex set $V(G) \neq \emptyset$, the edge set $E(G)$, and an incidence function $\psi_{G}$ that associates with each edge of $G$, an unordered pair of vertices of $G$. If $e$ is an edge and $u$ and $v$ are vertices such that $\psi_{G}(e)=u v$, then $e$ is said to join $u$ and $v$, and the vertices $u$ and $v$ are called the ends of $e$. The cardinality of $V(G)$ and $E(G)$ is denoted by $|G|$ and $|E(G)|$, respectively. We denote the degree and the neighborhood of a vertex $v$ of $G$ by $d_{G}(v)$ and $N_{G}(v)$; then $d_{G}(v)=\left|N_{G}(v)\right|$. As usual, the distance between vertices $u$ and $v$ of a connected graph $G$, denoted by $d_{G}(u, v)$, is defined as the number of edges in a shortest path connecting the vertices $u$ and $v$. Suppose that $d(x \mid G)=$ $\sum_{v \in V(G)} d_{G}(x, v)$ and $d^{2}(x \mid G)=\sum_{v \in V(G)}\left(d_{G}(x, v)\right)^{2}$. The eccentricity $\varepsilon_{G}(v)$ of a vertex $v$ in $G$ is the largest distance between $v$ and any other vertex $u$ of $G$; that is, $\varepsilon_{G}(v)=$ $\max _{u \in V(G)} d_{G}(u, v)$. For two graphs $G$ and $H$, if there exist two bijections $\theta: V(G) \rightarrow V(H)$ and $\varphi: E(G) \rightarrow E(H)$ such that $\psi_{G}(e)=u v$ if and only if $\psi_{H}(\varphi(e))=\theta(u) \theta(v)$, then we say that $G$ and $H$ are isomorphic, denoted by $G \cong H$. Let $\operatorname{Top}(G)$ denote a certain topological index of $G$. In general, if $G \cong H$, then $\operatorname{Top}(G)=\operatorname{Top}(H)$.

The total eccentricity and the eccentric connectivity indices $\zeta(G)$ and $\xi^{c}(G)$ of graph $G$ are defined as $\zeta(G)=$ $\sum_{v \in V(G)} \varepsilon_{G}(v)$ and $\xi^{c}(G)=\sum_{u v \in E(G)}\left[\varepsilon_{G}(u)+\varepsilon_{G}(v)\right]=$ $\sum_{v \in V(G)} d_{G}(v) \varepsilon_{G}(v)$, respectively. The Zagreb indices of $G$ are defined as $M_{1}(G)=\sum_{u v \in E(G)}\left[d_{G}(u)+d_{G}(v)\right]=$ $\sum_{u \in V(G)}\left(d_{G}(u)\right)^{2}$ and $M_{2}(G)=\sum_{u v \in E(G)} d_{G}(u) d_{G}(v)$. Very recently, the topological indices based on vertex eccentricities attracted some attention in chemistry. In an analogy with the Zagreb indices, the first and second Zagreb eccentricity indices $M_{1}^{*}$ and $M_{2}^{*}$ of a connected graph $G$ are defined by $[4,5]$. That is,

$$
\begin{gathered}
M_{1}^{*}(G)=\sum_{v \in V(G)} \varepsilon_{G}^{2}(v)=\sum_{v \in V(G)}\left(\varepsilon_{G}(v)\right)^{2}, \\
M_{2}^{*}(G)=\sum_{u v \in E(G)} \varepsilon_{G}(u) \varepsilon_{G}(v) .
\end{gathered}
$$

Lemma 1 (see [4]). Let $K_{n}$ be the complete graph of order $n$; then $\zeta\left(K_{n}\right)=n, \xi^{c}\left(K_{n}\right)=n(n-1), M_{1}^{*}\left(K_{n}\right)=n$, and $M_{2}^{*}\left(K_{n}\right)=\left(\begin{array}{c}n \\ 2\end{array}\right)$.

Lemma 2 (see [4]). Let $C_{n}$ be the cycle of length $n$; then $\zeta\left(C_{n}\right)=n\lfloor n / 2\rfloor, \xi^{c}\left(C_{n}\right)=2 n\lfloor n / 2\rfloor$, and $M_{1}^{*}\left(C_{n}\right)=M_{2}^{*}\left(C_{n}\right)=$ $n\lfloor n / 2\rfloor^{2}$.

Lemma 3 (see [4]). Let $P_{n}$ be the path on $n \geq 2$ vertices. Then

$$
\begin{gathered}
\zeta\left(P_{n}\right)= \begin{cases}\frac{1}{4} n(3 n-2), & 2 \mid n, \\
\frac{1}{4}(n-1)(3 n+1), & 2+n,\end{cases} \\
M_{1}^{*}\left(P_{n}\right)= \begin{cases}\frac{1}{12} n(n-1)(7 n-2), & 2 \mid n, \\
\frac{1}{12}(n-1)\left(7 n^{2}-2 n-3\right), & 2+n,\end{cases}
\end{gathered}
$$

$$
\begin{gathered}
\xi^{c}\left(P_{n}\right)= \begin{cases}\frac{1}{2}\left(3 n^{2}-6 n+4\right), & 2 \mid n, \\
\frac{3}{2}(n-1)^{2}, & 2+n,\end{cases} \\
M_{2}^{*}\left(P_{n}\right)= \begin{cases}\frac{1}{12} n\left(7 n^{2}-21 n+20\right), & 2 \mid n, \\
\frac{1}{12}(n-1)\left(7 n^{2}-14 n+3\right), & 2+n .\end{cases}
\end{gathered}
$$

\section{Zagreb Eccentricity Indices of Generalized Hierarchical Product Graphs}

In this section, we calculate the Zagreb eccentricity indices of the generalized hierarchical product graphs.

Definition 4 (see [9]). Let $G$ and $H$ be two connected graphs; $\emptyset \neq U \subseteq V(G)$. Then the generalized hierarchical product $G(U) \sqcap H$ is the graph with vertex set $V(G) \times V(H)$ and vertices $\left(u_{r}, v_{i}\right)$ and $\left(u_{s}, v_{k}\right)$ are adjacent if and only if $\left[u_{r}=u_{s} \in U\right.$ and $\left.v_{i} v_{k} \in E(H)\right]$ or $\left[v_{i}=v_{k} \in V(H)\right.$ and $\left.u_{r} u_{s} \in E(G)\right]$.

Given a connected graph $G$ and $\emptyset \neq U \subseteq V(G)$, a path connecting vertices $x$ and $y$ through $U$ is a $x U y$-path of $G$ containing some vertex $z \in U$ (vertex $z$ could be the vertex $x$ or vertex $y$ ). Then the distance through $U$ between $x$ and $y$ is denoted by $d_{G(U)}(x, y)$, which is the length of the shortest path $x U y$ in $G$. Note that if one of the vertex $x$ and $y$ belongs to $U$, then $d_{G(U)}(x, y)=d_{G}(x, y)$; see [13]. Similarly, we define some invariants related to $U$ in $G$ as follows:

$$
\begin{aligned}
& \varepsilon_{G(U)}(v)=\max _{u \in V(G)} d_{G(U)}(u, v), \\
& \zeta(G(U))=\sum_{v \in V(G)} \varepsilon_{G(U)}(v), \\
& \epsilon(G(U))=\sum_{v \in U} \varepsilon_{G(U)}(v) ; \text { see }[15], \\
& \xi^{c}(G(U))=\sum_{u v \in E(G)}\left(\varepsilon_{G(U)}(u)+\varepsilon_{G(U)}(v)\right) ; \text { see }[15], \\
& M_{1}^{*}(G(U))=\sum_{v \in V(G)} \varepsilon_{G(U)}^{2}(v), \\
& m_{1}^{*}(G(U))=\sum_{v \in U} \varepsilon_{G(U)}^{2}(v), \\
& M_{2}^{*}(G(U))=\sum_{u v \in E(G)} \varepsilon_{G(U)}(u) \varepsilon_{G(U)}(v) .
\end{aligned}
$$

Theorem 5 (see [15]). Let graphs $G$ and $H$ be connected; $\emptyset \neq U \subseteq V(G)$. Then

$$
\begin{aligned}
\xi^{c}(G(U) \sqcap H)= & |U| \xi^{c}(H)+|H| \xi^{c}(G(U)) \\
& +2|E(G)| \zeta(H)+2|E(H)| \epsilon(G(U)) .
\end{aligned}
$$

Lemma 6 (see [10]). Let $G$ and $H$ be two connected graphs and $\emptyset \neq U \subseteq V(G)$. Then

$$
\varepsilon_{G(U) \sqcap H}(u, v)=\varepsilon_{G(U)}(u)+\varepsilon_{H}(v) .
$$

Theorem 7. Let graphs $G$ and $H$ be connected; $\emptyset \neq U \subseteq V(G)$. Then

(i)

$$
\begin{aligned}
M_{1}^{*}(G(U) \sqcap H)= & |H| M_{1}^{*}(G(U)) \\
& +|G| M_{1}^{*}(H)+2 \zeta(G(U)) \zeta(H),
\end{aligned}
$$


(ii)

$$
\begin{aligned}
M_{2}^{*}(G(U) \sqcap H)= & |E(H)| m_{1}^{*}(G(U))+|E(G)| M_{1}^{*}(H) \\
& +|U| M_{2}^{*}(H)+|H| M_{2}^{*}(G(U)) \\
& +\xi^{c}(G(U)) \zeta(H)+\xi^{c}(H) \epsilon(G(U)) .
\end{aligned}
$$

Proof. Let $V(G)=\left\{u_{1}, u_{2}, \ldots, u_{n}\right\}$ and $V(H)=\left\{v_{1}, v_{2}, \ldots\right.$, $\left.v_{n^{\prime}}\right\}$.

(i) By the definition of Zagreb eccentricity index $M_{1}^{*}$ and Lemma 6, we have

$$
\begin{aligned}
M_{1}^{*}(G(U) \sqcap H)= & \sum_{\left(u_{r}, v_{i}\right) \in V(G(U) \sqcap H)} \varepsilon_{G(U) \sqcap H}^{2}\left(\left(u_{r}, v_{i}\right)\right) \\
= & \sum_{u_{r} \in V(G)} \sum_{v_{i} \in V(H)}\left(\varepsilon_{G(U)}\left(u_{r}\right)+\varepsilon_{H}\left(v_{i}\right)\right)^{2} \\
= & |H| \sum_{u_{r} \in V(G)} \varepsilon_{G(U)}^{2}\left(u_{r}\right)+|G| \sum_{v_{i} \in V(H)} \varepsilon_{H}^{2}\left(v_{i}\right) \\
& +2 \sum_{u_{r} \in V(G)} \varepsilon_{G(U)}\left(u_{r}\right) \sum_{v_{i} \in V(H)} \varepsilon_{H}\left(v_{i}\right) \\
= & |H| M_{1}^{*}(G(U))+|G| M_{1}^{*}(H) \\
& +2 \zeta(G(U)) \zeta(H) .
\end{aligned}
$$

(ii) We partition the edges of $G(U) \sqcap H$ into two subsets $E_{1}$ and $E_{2}$, as follows:

$$
\begin{aligned}
E_{1}= & \left\{\left(u_{r}, v_{i}\right)\left(u_{r}, v_{k}\right)\right. \\
& \left.\in E(G(U) \sqcap H) \mid v_{i} v_{k} \in E(H), u_{r} \in U\right\}, \\
E_{2}=\left\{\left(u_{r}, v_{i}\right)\left(u_{s}, v_{i}\right)\right. & \\
& \left.\in E(G(U) \sqcap H) \mid u_{r} u_{s} \in E(G), v_{i} \in V(H)\right\} .
\end{aligned}
$$

From the definition of Zagreb eccentricity index $M_{2}^{*}$ and Lemma 6, we get

$$
\begin{aligned}
M_{2}^{*}(G(U) \sqcap H) & \\
= & \sum_{\left(u_{r}, v_{i}\right)\left(u_{s}, v_{k}\right) \in E(G(U) \sqcap H)} \varepsilon_{G(U) \sqcap H}\left(\left(u_{r}, v_{i}\right)\right) \varepsilon_{G(U) \sqcap H}\left(\left(u_{s}, v_{k}\right)\right) \\
= & \sum_{\left(u_{r}, v_{i}\right)\left(u_{s}, v_{k}\right) \in E_{1} \cup E_{2}} \varepsilon_{G(U) \sqcap H}\left(\left(u_{r}, v_{i}\right)\right) \varepsilon_{G(U) \sqcap H}\left(\left(u_{s}, v_{k}\right)\right) \\
= & \sum_{u_{r} \in U} \sum_{v_{i} v_{k} \in E(H)}\left[\varepsilon_{G(U)}\left(u_{r}\right)+\varepsilon_{H}\left(v_{i}\right)\right]\left[\varepsilon_{G(U)}\left(u_{r}\right)+\varepsilon_{H}\left(v_{k}\right)\right] \\
& +\sum_{v_{i} \in V(H)} \sum_{u_{r} u_{s} \in E(G)}\left[\varepsilon_{G(U)}\left(u_{r}\right)+\varepsilon_{H}\left(v_{i}\right)\right] \\
& \times\left[\varepsilon_{G(U)}\left(u_{s}\right)+\varepsilon_{H}\left(v_{i}\right)\right]
\end{aligned}
$$

$$
\begin{aligned}
& =|E(H)| \sum_{u_{r} \in U} \varepsilon_{G(U)}^{2}\left(u_{r}\right)+\sum_{u_{r} \in U} \varepsilon_{G(U)}\left(u_{r}\right) \\
& \quad \times \sum_{v_{i} v_{k} \in E(H)}\left(\varepsilon_{H}\left(v_{i}\right)+\varepsilon_{H}\left(v_{k}\right)\right) \\
& +|U| \sum_{v_{i} v_{k} \in E(H)} \varepsilon_{H}\left(v_{i}\right) \varepsilon_{H}\left(v_{k}\right)+|E(G)| \sum_{v_{i} \in V(H)} \varepsilon_{H}^{2}\left(v_{i}\right) \\
& \quad+\sum_{v_{i} \in V(H)} \varepsilon_{H}\left(v_{i}\right) \sum_{u_{r} u_{s} \in E(G)}\left[\varepsilon_{G(U)}\left(u_{r}\right)+\varepsilon_{G(U)}\left(u_{s}\right)\right] \\
& +|H| \sum_{u_{r} u_{s} \in E(G)} \varepsilon_{G(U)}\left(u_{r}\right) \varepsilon_{G(U)}\left(u_{s}\right) \\
& =|E(H)| m_{1}^{*}(G(U))+\epsilon(G(U)) \xi^{c}(H)+|U| M_{2}^{*}(H) \\
& +|E(G)| M_{1}^{*}(H)+\zeta(H) \xi^{c}(G(U))+|H| M_{2}^{*}(G(U)) .
\end{aligned}
$$

This completes the proofs.

Definition 8 (see [13]). Let $G$ and $H$ be two connected graphs. Then the Cartesian product $G \square H$ has the vertex set $V(G) \times$ $V(H)$ and vertices $\left(u_{r}, v_{i}\right)$ and $\left(u_{s}, v_{k}\right)$ are adjacent if and only if $\left[u_{r}=u_{s} \in V(G)\right.$ and $\left.v_{i} v_{k} \in E(H)\right]$ or $\left[v_{i}=v_{k} \in V(H)\right.$ and $\left.u_{r} u_{s} \in E(G)\right]$, where $r, s=1,2, \ldots,|G|$ and $i, k=1,2, \ldots,|H|$.

Note that if $U=V(G)$, then $G(U) \sqcap H \cong G \square H$. So by Theorem 7 , the following corollary is obvious.

Corollary 9. Let $G$ and $H$ be two connected graphs. Then

$$
\begin{aligned}
M_{1}^{*}(G \square H)=\mid & |G| M_{1}^{*}(H)+|H| M_{1}^{*}(G)+2 \zeta(G) \zeta(H), \\
M_{2}^{*}(G \square H)= & |E(G)| M_{1}^{*}(H)+|E(H)| M_{1}^{*}(G) \\
& +|G| M_{2}^{*}(H)+|H| M_{2}^{*}(G) \\
& +\xi^{c}(G) \zeta(H)+\xi^{c}(H) \zeta(G) .
\end{aligned}
$$

Remark 10. Equation (14) corrects the corresponding Corollary 9 in [4]. According to (14), we recompute the second Zagreb eccentricity indices of the $C_{4}$ nanotubes as below (see Example 11).

Example 11. Using the results as above, it is easy to obtain the first and second Zagreb eccentricity indices of the $C_{4}$ nanotorus $C_{m} \square C_{n}(m \geq n \geq 3)$ and the $C_{4}$ nanotubes $P_{2 m} \square C_{n}(n \geq 3)$ and $P_{2 m+1} \square C_{n}(n \geq 3)$. By Corollary 9 and Lemmas 2 and 3 , we have

$$
\begin{aligned}
& M_{1}^{*}\left(C_{m} \square C_{n}\right)=\frac{1}{2} M_{2}^{*}\left(C_{m} \square C_{n}\right)=m n\left(\left\lfloor\frac{m}{2}\right\rfloor+\left\lfloor\frac{n}{2}\right\rfloor\right)^{2}, \\
& M_{1}^{*}\left(P_{2 m} \square C_{n}\right) \\
& \quad=m n\left[\frac{14}{3} m^{2}-3 m+\frac{1}{3}+2\left\lfloor\frac{n}{2}\right\rfloor^{2}+2(3 m-1)\left\lfloor\frac{n}{2}\right\rfloor\right],
\end{aligned}
$$




$$
\begin{aligned}
M_{1}^{*}\left(P_{2 m+1} \square C_{n}\right)= & m n\left(\frac{14}{3} m^{2}+4 m+\frac{1}{3}\right) \\
& +n(2 m+1)\left\lfloor\frac{n}{2}\right\rfloor^{2} \\
& +2 m n(3 m+2)\left\lfloor\frac{n}{2}\right\rfloor, \\
M_{2}^{*}\left(P_{2 m} \square C_{n}\right)= & m n\left(\frac{28}{3} m^{2}-10 m+\frac{11}{3}\right) \\
+ & (4 m-1) n\left\lfloor\frac{n}{2}\right\rfloor^{2} \\
+ & 2\left(6 m^{2}-4 m+1\right) n\left\lfloor\frac{n}{2}\right\rfloor, \\
M_{2}^{*}\left(P_{2 m+1}^{\left.\square C_{n}\right)=}\right. & m n\left(\frac{28}{3} m^{2}+4 m-\frac{1}{3}\right) \\
& +(4 m+1) n\left\lfloor\frac{n}{2}\right\rfloor^{2} \\
& +4 m(3 m+1) n\left\lfloor\frac{n}{2}\right\rfloor .
\end{aligned}
$$

Lemma 12 (see [19]). Let $\square_{i=1}^{n} G_{i}$ be Cartesian product of $n \geq 2$ connected graphs $G_{i}$. Then

(a) $\left|\square_{i=1}^{n} G_{i}\right|=\left|V\left(\square_{i=1}^{n} G_{i}\right)\right|=\prod_{i=1}^{n}\left|G_{i}\right|$.

(b) $\left|E\left(\square_{i=1}^{n} G_{i}\right)\right|=\sum_{i=1}^{n}\left[\prod_{j=1, j \neq i}^{n}\left|G_{j}\right|\left|E\left(G_{i}\right)\right|\right]=$ $\sum_{i=1}^{n}\left|E\left(G_{i}\right)\right| \prod_{j=1, j \neq i}^{n}\left|G_{j}\right|$.

(c) $\zeta\left(\square_{i=1}^{n} G_{i}\right)=\sum_{i=1}^{n}\left[\prod_{j=1, j \neq i}^{n}\left|G_{j}\right| \zeta\left(G_{i}\right)\right]=$ $\sum_{i=1}^{n} \zeta\left(G_{i}\right) \prod_{j=1, j \neq i}^{n}\left|G_{j}\right|$.

Corollary 13. Let $\square_{i=1}^{n} G_{i}$ be Cartesian product of $n \geq 2$ simple connected graphs $G_{i}$. Then

$$
\begin{aligned}
M_{1}^{*}\left(\square_{i=1}^{n} G_{i}\right)= & \sum_{i=1}^{n}\left[\prod_{j=1, j \neq i}^{n}\left|G_{j}\right| M_{1}^{*}\left(G_{i}\right)\right] \\
& +2 \sum_{1 \leq s<t \leq n}\left[\prod_{k=1, k \neq s, t}^{n}\left|G_{k}\right| \zeta\left(G_{s}\right) \zeta\left(G_{t}\right)\right] .
\end{aligned}
$$

Proof. The case $n=2$ is proved in Corollary 9. We prove the assertion by induction. Suppose the result is valid for $n$ graphs. Then by Lemma 12, we have

$$
\begin{aligned}
M_{1}^{*}\left(\square_{i=1}^{n+1} G_{i}\right)= & M_{1}^{*}\left(G_{n+1} \square\left(\square_{i=1}^{n} G_{i}\right)\right) \\
= & \left|G_{n+1}\right| M_{1}^{*}\left(\square_{i=1}^{n} G_{i}\right)+\left|\square_{i=1}^{n} G_{i}\right| M_{1}^{*}\left(G_{n+1}\right) \\
& +2 \zeta\left(G_{n+1}\right) \zeta\left(\square_{i=1}^{n} G_{i}\right) \\
= & \left|G_{n+1}\right| \sum_{i=1}^{n}\left[\prod_{j=1, j \neq i}^{n}\left|G_{j}\right| M_{1}^{*}\left(G_{i}\right)\right]+2\left|G_{n+1}\right|
\end{aligned}
$$

$$
\begin{aligned}
& \times \sum_{1 \leq s<t \leq n}\left[\prod_{k=1, k \neq s, t}^{n}\left|G_{k}\right| \zeta\left(G_{s}\right) \zeta\left(G_{t}\right)\right] \\
& +\prod_{i=1}^{n}\left|G_{i}\right| M_{1}^{*}\left(G_{n+1}\right) \\
& +2 \zeta\left(G_{n+1}\right) \sum_{i=1}^{n}\left[\prod_{j=1, j \neq i}^{n}\left|G_{j}\right| \zeta\left(G_{i}\right)\right] \\
& =\sum_{i=1}^{n+1}\left[\prod_{j=1, j \neq i}^{n+1}\left|G_{j}\right| M_{1}^{*}\left(G_{i}\right)\right] \\
& +2 \sum_{1 \leq s<t \leq n+1}\left[\prod_{k=1, k \neq s, t}^{n+1}\left|G_{k}\right| \zeta\left(G_{s}\right) \zeta\left(G_{t}\right)\right] .
\end{aligned}
$$

This completes the proof.

Example 14. The Hamming graph $H_{n_{1}, n_{2}, \ldots, n_{s}}=\square_{i=1}^{n} K_{n_{i}}$. Thus, by Corollary 13 and Lemma 1, we have $M_{1}^{*}\left(H_{n_{1}, n_{2}, \ldots, n_{s}}\right)=$ $s^{2} \prod_{i=1}^{s} n_{i}$. For $n_{1}=n_{2}=\cdots=n_{s}=2$, we attain the $s$ dimensional hypercubes $Q_{s}$. Therefore, $M_{1}^{*}\left(Q_{s}\right)=s^{2} 2^{s}$.

Remark 15. Ghorbani and Hosseinzadeh computed the second Zagreb eccentricity index of $\square_{i=1}^{n} G_{i}$ in [4]. Here, we can also obtain the explicit formula of $M_{2}^{*}\left(\square_{i=1}^{n} G_{i}\right)$ by induction.

For two connected graphs $G_{i}(i=1,2)$, we note that $G_{1}(U) \sqcap G_{2} \cong G_{1} \square G_{2}$ if $U=V\left(G_{1}\right)$; then by Theorem 5 , we have

$$
\begin{aligned}
\xi^{c}\left(G_{1} \square G_{2}\right)= & \left|G_{1}\right| \xi^{c}\left(G_{2}\right)+\left|G_{2}\right| \xi^{c}\left(G_{1}\right) \\
& +2\left|E\left(G_{1}\right)\right| \zeta\left(G_{2}\right)+2\left|E\left(G_{2}\right)\right| \zeta\left(G_{1}\right) .
\end{aligned}
$$

By induction, we can easily prove that

$$
\begin{aligned}
\xi^{c}\left(\square_{i=1}^{n} G_{i}\right)= & \sum_{i=1}^{n}\left[\prod_{j=1, j \neq i}^{n}\left|G_{j}\right| \xi^{c}\left(G_{i}\right)\right] \\
& +2 \sum_{\substack{1 \leq s, t \leq n \\
s \neq t}}\left[\prod_{\substack{k=1 \\
k \neq s, t}}^{n}\left|G_{k}\right|\left|E\left(G_{s}\right)\right| \zeta\left(G_{t}\right)\right] .
\end{aligned}
$$

Therefore, by Corollary 9, Lemma 12 and the formula as above, using a similar method of proof in Corollary 13, we can obtain Corollary 16.

Corollary 16 (see [4]). Let $\square_{i=1}^{n} G_{i}$ be Cartesian product of $n \geq$ 2 graphs $G_{i}$. Then

$$
\begin{aligned}
& M_{2}^{*}\left(\square_{i=1}^{n} G_{i}\right) \\
& =\sum_{\substack{1 \leq s, t \leq n \\
s \neq t}}\left[\prod_{\substack{k=1 \\
k \neq s, t}}^{n}\left|G_{k}\right|\left(\left|E\left(G_{s}\right)\right| M_{1}^{*}\left(G_{t}\right)+\xi^{c}\left(G_{s}\right) \zeta\left(G_{t}\right)\right)\right]
\end{aligned}
$$




$$
\begin{aligned}
+\sum_{i=1}^{n}[ & \prod_{\substack{j=1 \\
j \neq i}}^{n}\left|G_{j}\right| M_{2}^{*}\left(G_{i}\right) \\
& \left.+2 \sum_{\substack{1 \leq s<t \leq n \\
s, t \neq i}}\left(\prod_{\substack{k=1 \\
k \neq i, s, t}}^{n}\left|G_{j}\right| \zeta\left(G_{s}\right) \zeta\left(G_{t}\right)\right)\left|E\left(G_{i}\right)\right|\right] .
\end{aligned}
$$

From Corollaries 13 and 16, the following corollary is obvious.

Corollary 17. Let $G^{n}=\square_{i=1}^{n} G=\underbrace{G \square \cdots \square G}_{n \text { times }}$ be Cartesian product of $n \geq 2$ connected graphs $G$. Then

$$
\begin{aligned}
M_{1}^{*}\left(G^{n}\right) & =n|G|^{n-2}\left[|G| M_{1}^{*}(G)+(n-1) \zeta^{2}(G)\right], \\
M_{2}^{*}\left(G^{n}\right)= & 2\left(\begin{array}{c}
n \\
2
\end{array}\right)|G|^{n-2}\left[|E(G)| M_{1}^{*}(G)+\xi^{c}(G) \zeta(G)\right] \\
& +n|G|^{n-1} M_{2}^{*}(G)+2 n\left(\begin{array}{c}
n-1 \\
2
\end{array}\right)|G|^{n-3} \zeta^{2}(G) .
\end{aligned}
$$

\section{Zagreb Eccentricity Indices of $S$-Sum Graphs}

Let $G$ be a connected graph. The vertices of a Line graph $L(G)$ are the edges of $G$. Two edges of $G$ that share a vertex are considered to be adjacent in $L(G)$. A Subdivision graph $S(G)$ is the graph obtained by inserting an additional vertex in each edge of $G$. That is, each edge of $G$ is replaced by a path of length two.

Definition 18 (see [15]). For two connected graphs $G$ and $H$, the $S$-sum $G{ }_{S} H$ of $G$ and $H$ is a graph with vertex set $(V(G) \cup E(G)) \times V(H)$ and vertices $\left(u_{r}, v_{i}\right)$ and $\left(u_{s}, v_{k}\right)$ are adjacent if and only if $\left[u_{r}=u_{s} \in V(G)\right.$ and $\left.v_{i} v_{k} \in E(H)\right]$ or $\left[v_{i}=v_{k} \in V(H)\right.$ and $\left.u_{r} u_{s} \in E(S(G))\right]$.

Note that if $\emptyset \neq U=V(G) \subset V(S(G))$, then $G+{ }_{S} H \cong$ $S(G)(U) \sqcap H \cong S(G)(V(G)) \sqcap H$. So by Theorem 7, we can compute the Zagreb eccentricity indices of $G+{ }_{S} H$ easily.

Lemma 19 (see [15]). Let $G$ and $H$ be two connected graphs. If $U=V(G)$, then we have
(a) $|V(S(G))|=|G|+|E(G)|,|E(S(G))|=2|E(G)|$,
(b) for each vertex $v \in U$, we have $d_{S(G)}(v)=d_{G}(v)$,
(c) for each vertex $v \in V(S(G)) \backslash U$, we have $d_{S(G)}(v)=2$.

Lemma 20 (see [15]). Let $G=T_{n}(n \geq 2)$ be a tree with $n$ vertices. If $U=V(G)$, then

(a) for each vertex $v \in U$, we have $\varepsilon_{S(G)(U)}(v)=2 \varepsilon_{G}(v)$,

(b) for each vertex $v \in V(S(G)) \backslash U$, we have $\varepsilon_{S(G)(U)}(v)=$ $2 \varepsilon_{L(G)}(v)+1$.
Theorem 21. Let $T_{n}$ be a tree with order $n(n \geq 2)$ and let $H$ be a connected graph; $\emptyset \neq U=V\left(T_{n}\right) \subset V\left(S\left(T_{n}\right)\right)$. Then

(i)

$$
\begin{aligned}
M_{1}^{*}\left(T_{n}+{ }_{S} H\right)=\mid & |H|\left[4 M_{1}^{*}\left(T_{n}\right)+4 M_{1}^{*}\left(L\left(T_{n}\right)\right)\right. \\
& \left.+4 \zeta\left(L\left(T_{n}\right)\right)+n-1\right] \\
& +(2 n-1) M_{1}^{*}(H)+2 \zeta(H) \\
& \times\left[2 \zeta\left(T_{n}\right)+2 \zeta\left(L\left(T_{n}\right)\right)+n-1\right],
\end{aligned}
$$

(ii)

$$
\begin{aligned}
M_{2}^{*}\left(T_{n}+{ }_{S} H\right)= & 4|E(H)| M_{1}^{*}\left(T_{n}\right)+2(n-1) M_{1}^{*}(H) \\
& +n M_{2}^{*}(H)+2 \xi^{c}(H) \zeta\left(T_{n}\right) \\
& +2|H|\left[2 \rho\left(T_{n}\right)+\xi^{c}\left(T_{n}\right)\right] \\
& +2 \zeta(H)\left[\xi^{c}\left(T_{n}\right)+2 \zeta\left(L\left(T_{n}\right)\right)+n-1\right],
\end{aligned}
$$

where $\rho\left(T_{n}\right)=\sum_{u v \in E\left(T_{n}\right)} \varepsilon_{L\left(T_{n}\right)}(u v)\left[\varepsilon_{T_{n}}(u)+\varepsilon_{T_{n}}(v)\right]$.

Proof. (i) We start to calculate $M_{1}^{*}\left(T_{n}{ }_{S} H\right)$. By Lemmas 19 and 20, we have

$$
\begin{aligned}
M_{1}^{*}\left(S\left(T_{n}\right)(U)\right)= & \sum_{v \in V\left(S\left(T_{n}\right)\right)} \varepsilon_{S\left(T_{n}\right)(U)}^{2}(v) \\
= & 4 \sum_{v \in V\left(T_{n}\right)} \varepsilon_{T_{n}}^{2}(v)+\sum_{v \in V\left(L\left(T_{n}\right)\right)}\left[2 \varepsilon_{L\left(T_{n}\right)}(v)+1\right]^{2} \\
= & 4 M_{1}^{*}\left(T_{n}\right)+4 M_{1}^{*}\left(L\left(T_{n}\right)\right)+4 \zeta\left(L\left(T_{n}\right)\right) \\
& +n-1, \\
\zeta\left(S\left(T_{n}\right)(U)\right)= & \sum_{v \in V\left(S\left(T_{n}\right)\right)} \varepsilon_{S\left(T_{n}\right)(U)}(v) \\
= & 2 \sum_{v \in V\left(T_{n}\right)} \varepsilon_{T_{n}}(v)+2 \sum_{v \in V\left(L\left(T_{n}\right)\right)} \varepsilon_{L\left(T_{n}\right)}(v) \\
& +\left|E\left(T_{n}\right)\right| \\
= & 2 \zeta\left(T_{n}\right)+2 \zeta\left(L\left(T_{n}\right)\right)+n-1 .
\end{aligned}
$$

Combing these with (8) in Theorem 7, we can obtain the corresponding result.

(ii) Now, let us compute $M_{2}^{*}\left(T_{n}+{ }_{S} H\right)$. By Lemmas 19 and 20 , we get

$$
\begin{aligned}
m_{1}^{*}\left(S\left(T_{n}\right)(U)\right) & =\sum_{v \in U=V\left(T_{n}\right)} \varepsilon_{S\left(T_{n}\right)(U)}^{2}(v) \\
& =4 \sum_{v \in V\left(T_{n}\right)} \varepsilon_{T_{n}}^{2}(v)=4 M_{1}^{*}\left(T_{n}\right), \\
M_{2}^{*}\left(S\left(T_{n}\right)(U)\right) & =\sum_{u v \in E\left(T_{n}\right)} \varepsilon_{S\left(T_{n}\right)(U)}(u v)
\end{aligned}
$$




$$
\begin{aligned}
& \times\left[\varepsilon_{S\left(T_{n}\right)(U)}(u)+\varepsilon_{S\left(T_{n}\right)(U)}(v)\right] \\
& =2 \sum_{u v \in E\left(T_{n}\right)}\left[2 \varepsilon_{L\left(T_{n}\right)}(u v)+1\right] \\
& \times\left[\varepsilon_{T_{n}}(u)+\varepsilon_{T_{n}}(v)\right] \\
& =4 \sum_{u v \in E\left(T_{n}\right)} \varepsilon_{L\left(T_{n}\right)}(u v)\left[\varepsilon_{T_{n}}(u)+\varepsilon_{T_{n}}(v)\right] \\
& +2 \sum_{u v \in E\left(T_{n}\right)}\left[\varepsilon_{T_{n}}(u)+\varepsilon_{T_{n}}(v)\right] \\
& =4 \rho\left(T_{n}\right)+2 \xi^{c}\left(T_{n}\right), \\
& \xi^{c}\left(S\left(T_{n}\right)(U)\right)=\sum_{u v \in E\left(S\left(T_{n}\right)\right)}\left[\varepsilon_{S\left(T_{n}\right)(U)}(u)+\varepsilon_{S\left(T_{n}\right)(U)}(v)\right] \\
& =\sum_{v \in V\left(S\left(T_{n}\right)\right)} d_{S\left(T_{n}\right)(U)}(v) \varepsilon_{S\left(T_{n}\right)(U)}(v) \\
& =2 \sum_{v \in U=V\left(T_{n}\right)} d_{T_{n}}(v) \varepsilon_{T_{n}}(v) \\
& +2 \sum_{v \in V\left(L\left(T_{n}\right)\right)}\left(2 \varepsilon_{L\left(T_{n}\right)}(v)+1\right) \\
& =2 \xi^{c}\left(T_{n}\right)+4 \zeta\left(L\left(T_{n}\right)\right)+2(n-1), \\
& \epsilon\left(S\left(T_{n}\right)(U)\right)=\sum_{v \in U=V\left(T_{n}\right)} \varepsilon_{S\left(T_{n}\right)(U)}(v) \\
& =2 \sum_{v \in V\left(T_{n}\right)} \varepsilon_{T_{n}}(v)=2 \zeta\left(T_{n}\right) .
\end{aligned}
$$

Combing these results with (9) in Theorem 7, we obtain the desired result.

Clearly, if $n$ is even, then $\rho\left(P_{n}\right)=(1 / 2) n(n-2)+$ $2 \sum_{i=(n / 2)}^{n-2} i(2 i+1)$. Otherwise, if $n$ is odd, then $\rho\left(P_{n}\right)=$ $2 \sum_{i=((n-1) / 2)}^{n-2} i(2 i+1)$. So, the following lemma holds.

Lemma 22. Let $P_{n}$ be a path of order $n$. Then

$$
\rho\left(P_{n}\right)= \begin{cases}\frac{1}{12}(n-2)\left(14 n^{2}-23 n+12\right), & 2 \mid n, \\ \frac{1}{12}(n-1)\left(14 n^{2}-37 n+21\right), & 2+n .\end{cases}
$$

Example 23. Suppose $L_{n}$ is a linear hexagonal chain with $n \geq$ 2 hexagons (see Figure 1); note that $L_{n} \cong P_{n+1}+{ }_{S} P_{2}$. Thus, by Lemmas 3 and 22 and Theorem 21, we have

$$
M_{1}^{*}\left(L_{n}\right)=\frac{2}{3}\left(14 n^{3}+30 n^{2}+19 n+3\right)
$$

$$
M_{2}^{*}\left(L_{n}\right)= \begin{cases}\frac{1}{3}\left(35 n^{3}+57 n^{2}+25 n+3\right), & 2 \mid n, \\ \frac{1}{3}\left(35 n^{3}+57 n^{2}+28 n+6\right), & 2+n .\end{cases}
$$

Let $n$ be an integer with $n \geq 3$ and $U=V\left(C_{n}\right)$. Note that $\varepsilon_{S\left(C_{n}\right)(U)}(v)=n$ for any vertex $v \in V\left(S\left(C_{n}\right)\right)$. Then by Theorem 7 , we can obtain the following theorem.

Theorem 24. Let $C_{n}(n \geq 3)$ be a cycle and let $H$ be an arbitrary connected graph; $\emptyset \neq U=V\left(C_{n}\right) \subset V\left(S\left(C_{n}\right)\right)$. Then

$$
\begin{aligned}
& M_{1}^{*}\left(C_{n}{ }_{S} H\right)=2 n\left(n^{2}|H|+M_{1}^{*}(H)+2 n \zeta(H)\right), \\
& M_{2}^{*}\left(C_{n}+{ }_{S} H\right)=n[(|E(H)|+2|H|) n^{2} \\
&+\left(4 \zeta(H)+\xi^{c}(H)\right) n \\
&\left.+2 M_{1}^{*}(H)+M_{2}^{*}(H)\right] .
\end{aligned}
$$

Example 25. Let $n$ be an integer with $n \geq 3$ and let $\Gamma$ be the zigzag polyhex nanotube $\mathrm{TUHC}_{6}[2 n, 2]$ (see Figure 2); then $\Gamma \cong C_{n}+{ }_{S} P_{2}$. By Theorem 24 and Lemma 3 , we have $M_{1}^{*}(\Gamma)=$ $4 n(n+1)^{2}$ and $M_{2}^{*}(\Gamma)=5 n(n+1)^{2}$.

\section{Zagreb Eccentricity Indices of Cluster and Corona Product Graphs}

The cluster product, corona product, and join of two graphs are important graph operations defined as below.

Definition 26 (see [20]). The cluster product graph $G\{H\}$ is obtained by taking one copy of $G$ and $|G|$ copies of a rooted graph $H$ and by identifying the root of the $i$ th copy of $H$ with the $i$ th vertex of $G, i=1,2, \ldots,|G|$.

Definition 27 (see [20]). The corona product graph $G \odot H$ is obtained by taking one copy of $G$ and $|G|$ copies of $H$ and by joining each vertex of the $i$ th copy of $H$ to the $i$ th vertex of $G$, $i=1,2, \ldots,|G|$.

Definition 28 (see [20]). The join graph $G+H: V(G+H)=$ $V(G) \cup V(H) ; E(G+H)=E(G) \cup E(H) \cup\{(u, v) \mid u \in V(G), v \in$ $V(H)\}$.

Let $G$ and $H$ be connected graphs; $x$ is a root-vertex of $H$. Note that; if $\emptyset \neq U=\{x\} \subset V(H)$, then $G\{H\} \cong H(U) \sqcap G \cong H(\{x\}) \sqcap G$. We define $\omega_{x}(H)=$ $\sum_{u v \in E(H)} d_{H}(u, x) d_{H}(v, x)$ and $\varsigma_{x}(H)=\sum_{u v \in E(H)}\left[d_{H}(u, x)+\right.$ $\left.d_{H}(v, x)\right]=\sum_{v \in V(H)} d_{H}(v) d_{H}(v, x)$.

Theorem 29. Let $G$ and $H$ be two connected graphs; $x$ is a root-vertex of $H$. Then

$$
\begin{aligned}
\xi^{c}(G\{H\})= & \xi^{c}(G)+2|E(H)| \zeta(G) \\
& +2[|E(G)|+|G||E(H)|] \varepsilon_{H}(x)+|G| \varsigma_{x}(H),
\end{aligned}
$$




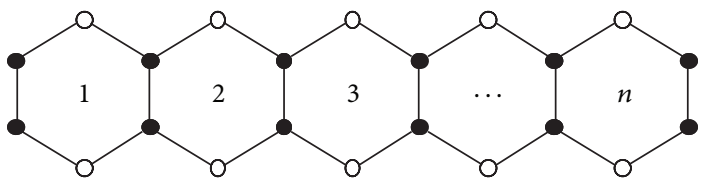

FIgURE 1: The linear hexagonal chain with $n$ hexagons $L_{n}$.

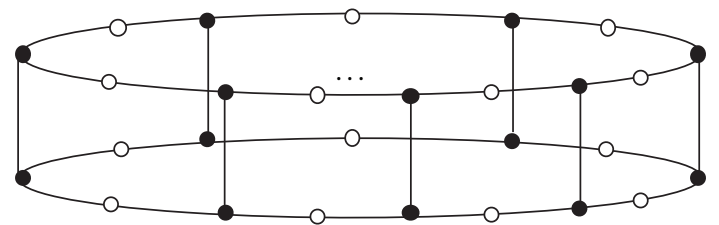

Figure 2: The zig-zay polyhex nanotube $\mathrm{TUHC}_{6}[2 n, 2]$.

$$
\begin{aligned}
M_{1}^{*}(G\{H\})= & |H| M_{1}^{*}(G) \\
+ & 2\left[|H| \varepsilon_{H}(x)+d(x \mid H)\right] \zeta(G)+|G| \\
& \times\left[|H| \varepsilon_{H}^{2}(x)+2 \varepsilon_{H}(x) d(x \mid H)\right. \\
& \left.+d^{2}(x \mid H)\right], \\
M_{2}^{*}(G\{H\})= & \varepsilon_{H}(x) \xi^{c}(G)+q^{\prime} M_{1}^{*}(G) \\
& +M_{2}^{*}(G)+\left[\varsigma_{x}(H)+2|E(H)| \varepsilon_{H}(x)\right] \zeta(G) \\
& +[|E(G)|+|G||E(H)|] \varepsilon_{H}^{2}(x) \\
& +|G| \varepsilon_{H}(x) \varsigma_{x}(H)+|G| \omega_{x}(H) .
\end{aligned}
$$

Proof. Let $\emptyset \neq U=\{x\} \subset V(H)$. Then

$$
\begin{aligned}
\xi^{c}(H(U)) & =\xi^{c}(H(\{x\}))=\sum_{u v \in E(H)}\left[\varepsilon_{H(\{x\})}(u)+\varepsilon_{H(\{x\})}(v)\right] \\
& =\sum_{u v \in E(H)}\left[d_{H}(u, x)+d_{H}(u, x)+2 \varepsilon_{H}(x)\right] \\
& =\varsigma_{x}(H)+2|E(H)| \varepsilon_{H}(x) .
\end{aligned}
$$

On the other hand, $\epsilon(H(U))=\epsilon(H(\{x\}))=\sum_{v=x} \varepsilon_{H(\{x\})}(v)=$ $\varepsilon_{H}(x)$. Note that $G\{H\} \cong G \sqcap H(\{x\})$. Thus, combing these results with Theorem 5 , we can obtain (29).

Similarly, we can determine (30) and (31) in terms of Theorem 7, respectively.

Let $G$ and $H$ be two simple graphs. If $|G|=n$ and $|E(G)|=$ $q$, then we say that $G$ is an $(n, q)$-graph. According to the definitions of the cluster and corona products, if $G$ is an $(n, q)$ graph and $H$ is a $\left(n^{\prime}, q^{\prime}\right)$-graph, then $G\{H\}$ is an $\left(n n^{\prime}, q+n q^{\prime}\right)$ graph and $G \odot H$ is an $\left(n n^{\prime}+n, q+n q^{\prime}+n n^{\prime}\right)$-graph.
Corollary 30. Let $G$ be a connected $(n, q)$-graph and $H$ is an arbitrary $\left(n^{\prime}, q^{\prime}\right)$-graph. Then

$$
\begin{aligned}
& \xi^{c}(G \odot H)=\xi^{c}(G)+2\left(q^{\prime}+n^{\prime}\right) \zeta(G)+2 q+4 n q^{\prime}+3 n n^{\prime}, \\
& M_{1}^{*}(G \odot H)=\left(n^{\prime}+1\right) M_{1}^{*}(G) \\
&+2\left(2 n^{\prime}+1\right) \zeta(G)+n\left(4 n^{\prime}+1\right), \\
& M_{2}^{*}(G \odot H)= \xi^{c}(G)+\left(n^{\prime}+q^{\prime}\right) M_{1}^{*}(G)+M_{2}^{*}(G) \\
&+\left(3 n^{\prime}+4 q^{\prime}\right) \zeta(G)+2 n\left(n^{\prime}+2 q^{\prime}\right)+q .
\end{aligned}
$$

Proof. For any $\left(n^{\prime}, q^{\prime}\right)$-graph $H$, let $x$ be the root-vertex of graph $H+x$ (the join of graphs $H$ and $K_{1}, x$ is the unique vertex in $\left.K_{1}\right)$. Then $H+x$ is a $\left(n^{\prime}+1, n^{\prime}+q^{\prime}\right)$-graph. It is easy to see that $\varepsilon_{H+x}(x)=1, \varsigma_{x}(H+x)=n^{\prime}+2 q^{\prime}$. Note that $G \odot H \cong G\{H+x\}$; hence $\xi^{c}(G \odot H)=\xi^{c}(G\{H+x\})$. Equation (33) is obtained by (29). Moreover, we note that $d(x \mid H+x)=d^{2}(x \mid H+x)=n^{\prime}$ and $\omega_{x}(H+x)=q^{\prime}$. Hence, by (30) and (31), using the same method as above, the corresponding equations (34) and (35) are also obtained, respectively.

As applications, we present some examples as below, these results can be attained by means of Corollary 30, Lemmas 2, and 3.

Example 31. The following equations hold:

$$
\begin{aligned}
& \xi^{c}\left(P_{m} \odot P_{n}\right)= \begin{cases}3 m^{2} n+5 m n-4 m, & 2 \mid m, m \geq 2 ; \\
3 m^{2} n+5 m n-4 m-n, & 2 \nmid m, m \geq 3,\end{cases} \\
& \xi^{c}\left(C_{m} \odot C_{n}\right)=m\left(2(2 n+1)\left\lfloor\frac{m}{2}\right\rfloor+7 n+2\right), \\
& m \geq 3, n \geq 3 \text {, } \\
& \xi^{c}\left(P_{m} \odot C_{n}\right) \\
& =\left\{\begin{array}{l}
\frac{1}{2}\left(6 m^{2} n+3 m^{2}+10 m n-2 m\right), \\
2 \mid m, m \geq 2, n \geq 3 ; \\
\frac{1}{2}\left(6 m^{2} n+3 m^{2}+10 m n-2 m-2 n-1\right), \\
2+m, m \geq 3, n \geq 3,
\end{array}\right. \\
& \xi^{c}\left(C_{m} \odot P_{n}\right)=m\left(4 n\left\lfloor\frac{m}{2}\right\rfloor+7 n-2\right), \quad m \geq 3 \text {. }
\end{aligned}
$$

Example 32. Let $H$ be an arbitrary graph with $n$ vertices. Then

$$
\begin{aligned}
& M_{1}^{*}\left(P_{m} \odot H\right) \\
& =\left\{\begin{array}{r}
\frac{1}{12} m\left(7 m^{2} n+7 m^{2}+27 m n+9 m+26 n+2\right), \\
2 \mid m, m \geq 2 ; \\
\frac{1}{12}\left(7 m^{3} n+7 m^{3}+27 m^{2} n+9 m^{2}-m-9 n-3\right), \\
2+m, m \geq 3,
\end{array}\right.
\end{aligned}
$$




$$
\begin{aligned}
& M_{1}^{*}\left(C_{m} \odot H\right) \\
& \quad=m\left((n+1)\left\lfloor\frac{m}{2}\right\rfloor^{2}+2(2 n+1)\left\lfloor\frac{m}{2}\right\rfloor+4 n+1\right),
\end{aligned}
$$$$
m \geq 3 \text {. }
$$

Example 33. The following equations hold:

$$
\begin{aligned}
& M_{2}^{*}\left(P_{m} \odot P_{n}\right)
\end{aligned}
$$

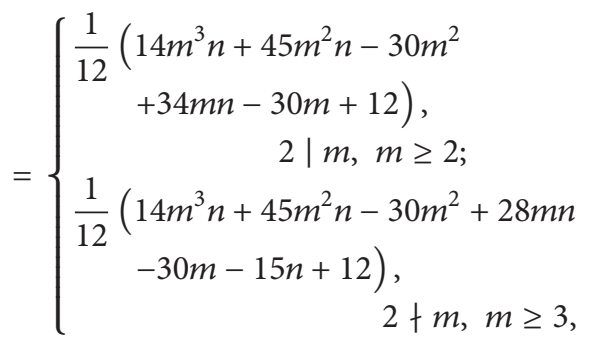

$$
\begin{aligned}
& M_{2}^{*}\left(C_{m} \odot C_{n}\right) \\
& =m\left((2 n+1)\left\lfloor\frac{m}{2}\right\rfloor^{2}+(7 n+2)\left\lfloor\frac{m}{2}\right\rfloor+6 n+1\right), \\
& m \geq 3, n \geq 3, \\
& M_{2}^{*}\left(P_{m} \odot C_{n}\right)
\end{aligned}
$$

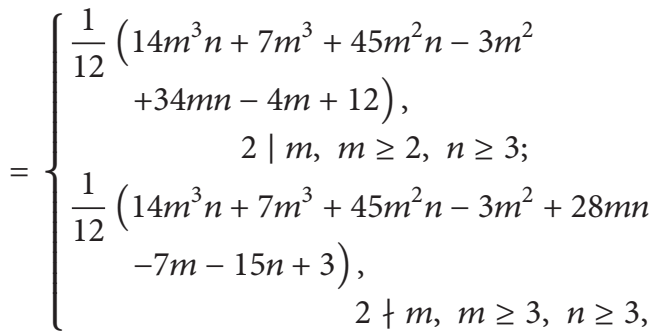

$$
\begin{aligned}
& M_{2}^{*}\left(C_{m} \odot P_{n}\right) \\
& =m\left(2 n\left\lfloor\frac{m}{2}\right\rfloor^{2}+(7 n-2)\left\lfloor\frac{m}{2}\right\rfloor+6 n-3\right), \\
& m \geq 3 \text {. }
\end{aligned}
$$

\section{Conflict of Interests}

The authors declare that there is no conflict of interests regarding the publication of this paper.

\section{Acknowledgments}

The authors would like to thank the referee for many helpful comments and suggestions, which have greatly improved the presentation of the paper. This work is supported by the National Natural Science Foundation of China (no. 11271006), the Scientific Research Programs of the Higher Education Institution of Xinjiang (XJEDU2012I37,I38), and the Science Foundation of Changji University (2012YJYB003).

\section{References}

[1] I. Gutman and O. E. Polansky, Mathematical Concepts in Organic Chemistry, Springer, Berlin, Germany, 1986.

[2] H. Wiener, "Structural determination of paraffin boiling points," Journal of the American Chemical Society, vol. 69, no. 1, pp. 1720, 1947.

[3] I. Gutman and N. Trinajstić, "Graph theory and molecular orbitals. Total $\varphi$-electron energy of alternant hydrocarbons," Chemical Physics Letters, vol. 17, no. 4, pp. 535-538, 1972.

[4] M. Ghorbani and M. A. Hosseinzadeh, "A new version of Zagreb indices," Filomat, vol. 26, no. 1, pp. 93-100, 2012.

[5] D. Vukičević and A. Graovac, "Note on the comparison of the first and second normalized Zagreb eccentricity indices," Acta Chimica Slovenica, vol. 57, no. 3, pp. 524-528, 2010.

[6] K. Ch. Das, D.-W. Lee, and A. Graovac, "Some properties of the Zagreb eccentricity indices," Ars Mathematica Contemporanea, vol. 6, no. 1, pp. 117-125, 2013.

[7] Z. Du, B. Zhou, and N. Trinajstić, "Extremal properties of the Zagreb eccentricity indices," Croatica Chemica Acta, vol. 85, no. 3, pp. 359-362, 2012.

[8] R. Xing, B. Zhou, and N. Trinajstić, "On zagreb eccentricity indices," Croatica Chemica Acta, vol. 84, no. 4, pp. 493-497, 2011.

[9] L. Barrière, F. Comellas, C. Dalfó, and M. A. Fiol, "The hierarchical product of graphs," Discrete Applied Mathematics, vol. 157, no. 1, pp. 36-48, 2009.

[10] L. Barrière, C. Dalfó, M. A. Fiol, and M. Mitjana, "The generalized hierarchical product of graphs," Discrete Mathematics, vol. 309, no. 12, pp. 3871-3881, 2009.

[11] M. Arezoomand and B. Taeri, "Applications of generalized hierarchical product of graphs in computing the Szeged index of chemical graphs," MATCH. Communications in Mathematical and in Computer, vol. 64, no. 3, pp. 591-602, 2010.

[12] M. Arezoomand and B. Taeri, "Zagreb indices of the generalized hierarchical product of graphs," MATCH. Communications in Mathematical and in Computer, vol. 69, no. 1, pp. 131-140, 2013.

[13] M. Eliasi and A. Iranmanesh, "The hyper-Wiener index of the generalized hierarchical product of graphs," Discrete Applied Mathematics, vol. 159, no. 8, pp. 866-871, 2011.

[14] M. Eliasi and A. Iranmanesh, "Hosoya polynomial of hierarchical product of graphs," MATCH. Communications in Mathematical and in Computer, vol. 69, no. 1, pp. 111-119, 2013.

[15] B. Eskender and E. Vumar, "Eccentric connectivity index and eccentric distance sum of some graph operations," Transactions on Combinatorics, vol. 2, no. 1, pp. 103-111, 2013.

[16] K. Pattabiraman and P. Paulraja, "Vertex and edge PadmakarIvan indices of the generalized hierarchical product of graphs," Discrete Applied Mathematics, vol. 160, no. 9, pp. 1376-1384, 2012.

[17] M. Tavakoli, F. Rahbarnia, and A. R. Ashrafi, "Further results on hierarchical product of graphs," Discrete Applied Mathematics, vol. 161, no. 7-8, pp. 1162-1167, 2013.

[18] D. B. West, Introduction to Graph Theory, Prentice Hall, Upper Saddle River, NJ, USA, 2nd edition, 1996.

[19] M. H. Khalifeh, H. Yousefi-Azari, and A. R. Ashrafi, "Vertex and edge PI indices of Cartesian product graphs," Discrete Applied Mathematics, vol. 156, no. 10, pp. 1780-1789, 2008.

[20] Y. N. Yeh and I. Gutman, "On the sum of all distances in composite graphs," Discrete Mathematics, vol. 135, no. 1-3, pp. 359365, 1994. 


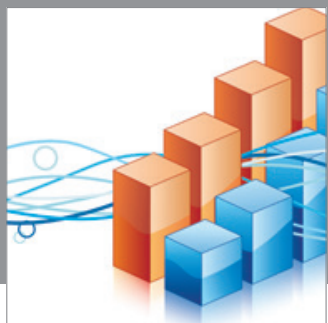

Advances in

Operations Research

mansans

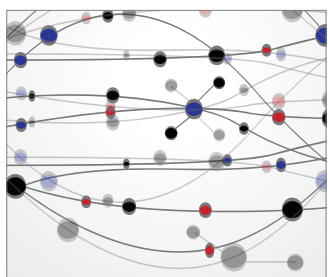

The Scientific World Journal
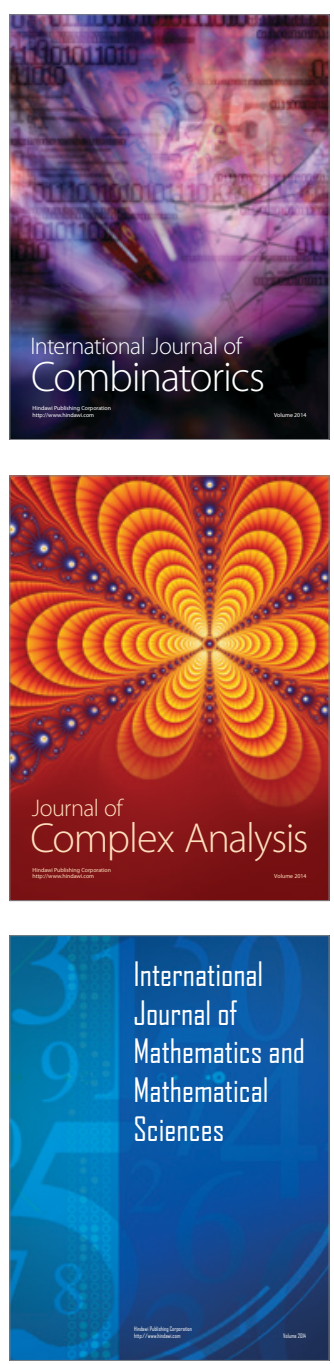
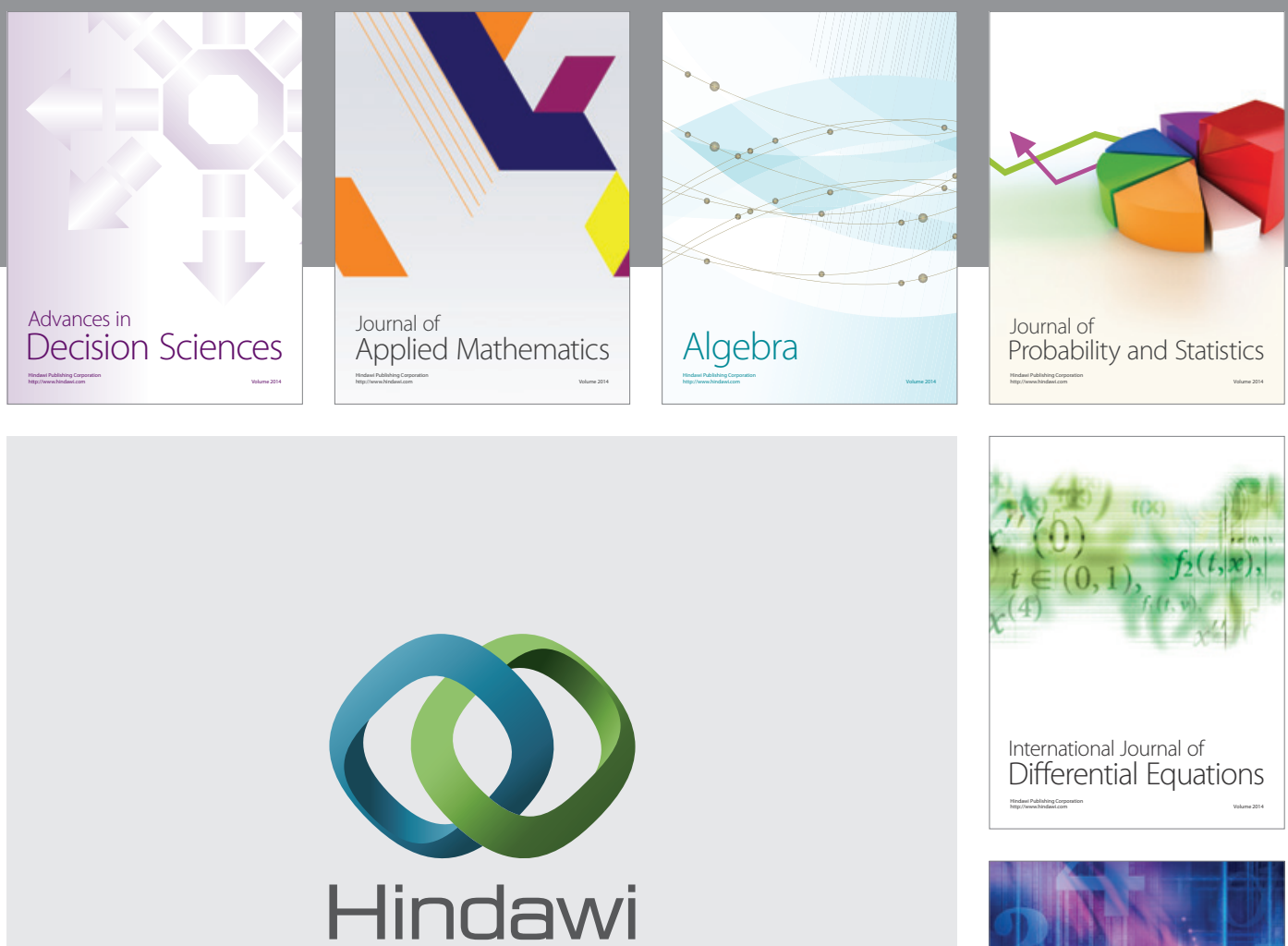

Submit your manuscripts at http://www.hindawi.com
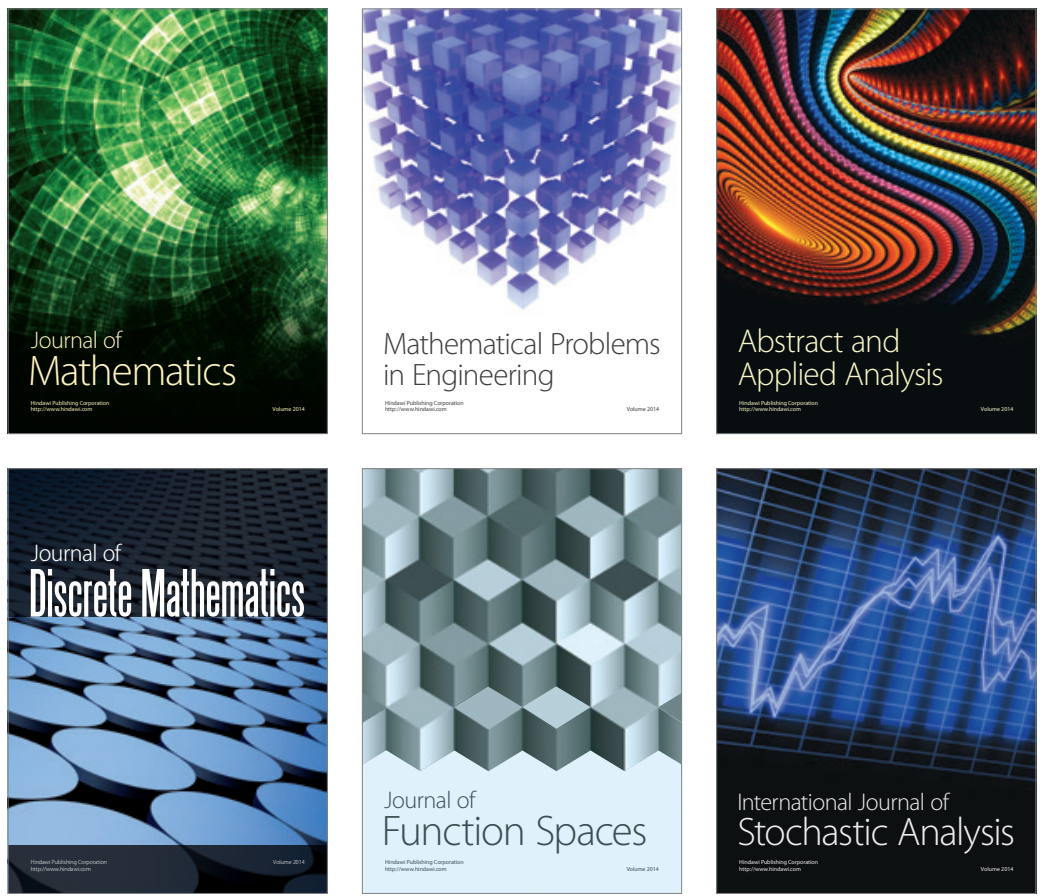

Journal of

Function Spaces

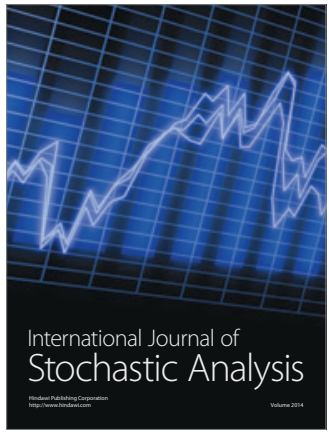

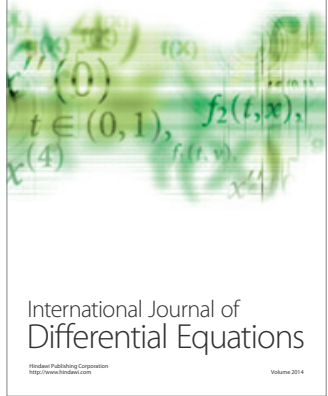
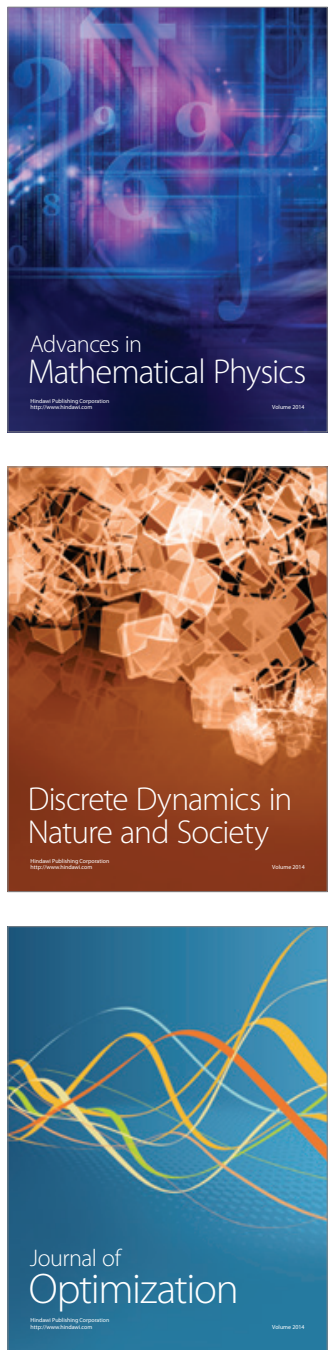